\title{
Effect of Sample Rate on saECG Spectrum
}

\author{
Jacob Melgaard, Claus Graff, Peter L Sørensen, Kasper Sørensen, \\ Samuel E Schmidt, Johannes J Struijk
}

Aalborg University, Aalborg, Denmark

\begin{abstract}
Signal-averaged ECG (saECG) has been used to show delayed ventricular activation and myocardial infarction, but alignment jitter acts as a low-pass filter. Jitter arises due to sampling of the signal, and due to imperfect alignment algorithms. In this study we investigated the influence of the sample rate on the saECG spectrum, i.e. if the jitter can be minimized by increasing the sample rate.

High speed high resolution ECGs were recorded from 6 volunteers in a shielded room. The spectrum of both raw beats and the saECG were computed and compared, and we found that sample rate does indeed influence the intrinsic low-pass filtering of the signal caused by jitter.
\end{abstract}

\section{Introduction}

Signal-averaged ECG has been used to show delayed ventricular activation caused by myocardial infarction. In short, the normal procedure is to transform the ECG to the $\mathrm{X}, \mathrm{Y}$ and Z VCG leads, align beats, make an average, and then high pass filter the average beats with cutoff of $25 \mathrm{~Hz}$ or $40 \mathrm{~Hz}$. In order not to have filter artefacts, the filtering is done towards the middle of the QRS complex, i.e. the first part of the signal is filtered ordinarily, while the last part is filtered backwards.

The critical part in this process is the alignment of each beat. "Comprehensive Electrocardiology" outlines that misalignment with a normal distribution and standard deviation of $1 \mathrm{~ms}$, corresponds to a low pass filter with cutoff of $140 \mathrm{~Hz}$ [1]. Such misalignment is termed jitter, and can of course have other distributions as well. State of the art alignment methods typically employ correlationbased template matching in an iterative fashion. Other methods such as dynamic time warping has also been used [2], but since they also change the frequency spectrum, they will not be considered further in this work. Although template matching is generally accepted to perform very well, there is no knowledge of the resulting jitter distribution.
If we consider the ECG mathematically, it can be written as:

$$
x(t)=\sum_{i} s_{i}\left(t+\delta_{i}\right)+v_{i}(t)
$$

where $x(t)$ is the entire signal, $s_{i}\left(t+\delta_{i}\right)$ is the $i$ th beat with some jitter $\delta_{i}$, and $v_{i}(t)$ is a noise term. First, in this case we assume that the noise is white Gaussian with low amplitude, and thus only adds a constant to the spectrum. Hence it can be neglected. Further, if we assume that each beat is identical (which is the underlying assumption for making a signal average), the averaged signal can be written as

$$
\overline{x(t)}=\frac{1}{N} \sum_{i=1}^{N} s\left(t+\delta_{i}\right) .
$$

The time-shift corresponds to a phase-shift in the frequency domain; the Fourier transform of $\overline{x(t)}$ is

$$
\boldsymbol{F}\{\overline{x(t)}\}=\left(\frac{1}{N} \sum_{i=1}^{N} e^{-j \omega \delta_{i}}\right) \boldsymbol{F}\left\{s\left(t+\delta_{i}\right)\right\}
$$

The transfer function can be found as the expected value of the term in parenthesis on the right hand side, which is dependent on the distribution of $\delta_{i}$. For normally distributed jitter with standard deviation $\sigma$ the expression for the cut-off frequency becomes

$$
f_{c}=\frac{\sqrt{\ln 2}}{2 \pi \sigma} \approx \frac{0.133}{\sigma},
$$

while for a uniform distribution (on the interval $\Delta$ ) we have

$$
\operatorname{sinc}\left(\omega_{c} \Delta\right)=\sqrt{2} \Leftrightarrow f_{c} \approx \frac{0.22}{\Delta}, \omega_{c}=2 \pi f_{c} .
$$

The aim of this study was twofold. Firstly, it was to investigate the effect of sample rate on the saECG spectrum. This is because sampling introduces uniformly distributed jitter with $\Delta=T=1 / F_{S}$. Secondly, it was to estimate any additional low-pass effect of the alignment algorithm in addition to the sampling-induced jitter.

\section{Methods}

High speed, high resolution 12-lead ECGs (8000 samples per second, 24 bit resolution) were recorded from 6 healthy volunteers in a shielded lab. Due to limitations in the recording device, three segments of 50 second duration 

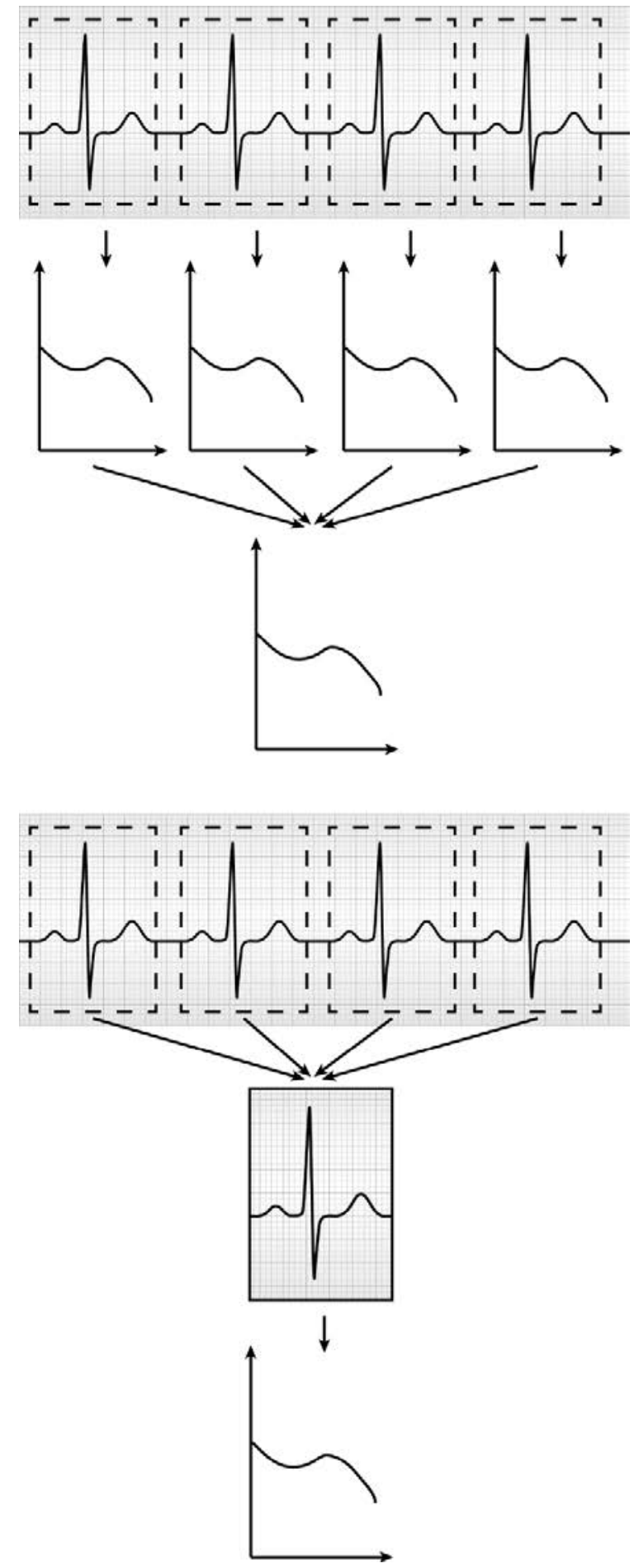

Figure 1. Illustration of how the two spectra that are being compared are computed.

Top panel: To estimate the actual spectrum, the spectrum of each beat is computed, and the average is taken.

Bottom panel: To obtain the saECG spectrum, the mean beat is computed, and then the spectrum. were recorded and stitched together to constitute one $150 \mathrm{~s}$ recording.

To avoid any loss of high-frequency signal content, no transformation to $\mathrm{X}, \mathrm{Y}$ and $\mathrm{Z}$ was made. Instead, leads V1 and V5 were used. Beats were segmented using an automatic iterative correlation-based template matching algorithm; only beats with $r>0.99$ were included in the analyses. To estimate the frequency content of one beat (denoted $s(t)$ above), the Fourier transform of each beat was made, and an average of these spectra was made. The saECG spectrum was found from the mean beat (see fig. 1). Finally, the difference between these two spectra was computed. This process was repeated for downsampled replicas of the recorded signal as well, sample rates of $4000 \mathrm{~Hz}, 2000 \mathrm{~Hz}, 1000 \mathrm{~Hz}$ and $500 \mathrm{~Hz}$ were obtained by simply taking every $2^{\text {nd }}, 4^{\text {th }}, 8^{\text {th }}$ and $16^{\text {th }}$ sample of the original signal.

\section{Results}

Attenuation at different selected frequencies are shown in table 1 (for lead V1) and table 2 (for lead V5). For both leads, there is a clear attenuation of the saECG spectrum compared to the estimated actual beat spectrum. The effect is larger in V1 than in V5, especially for higher frequencies. There is also a larger spread between subjects in V1 than in V5. If alignment was accurate to within one sample (and the underlying beats were completely identical), the $-3 \mathrm{~dB}$ frequencies are as given in table 3 . The attenuations found are much larger than these values - or, conversely, the actual low-pass cutoff is at a lower frequency than expected.

Looking at attenuation of frequencies up to $1000 \mathrm{~Hz}$ (typically up to $250 \mathrm{~Hz}$ is included in saECG analyses), there is a clear dependence between sample rate and attenuation. Higher sample rates result in lower attenuation.

Table 3. Expected cutoff frequency due to uniform sample jitter.

\begin{tabular}{cc}
\hline$F_{S}[\mathrm{~Hz}]$ & $f_{c}[\mathrm{~Hz}]$ \\
\hline 8000 & 1760 \\
4000 & 880 \\
2000 & 440 \\
1000 & 220 \\
500 & 110 \\
\hline
\end{tabular}

\section{Discussion}

The dependence between sample rate and attenuation clearly shows, that a large part of the attenuation is caused by sampling jitter. However, since the attenuation is larger than that caused only by sampling, other factors must affect the spectrum as well. One obvious factor is the reduced 
Table 1: Attenuation [dB] at select frequencies in lead V1 $(n=6)$.

\begin{tabular}{lrrrrrr}
\hline$F_{S}[\mathrm{~Hz}]$ & $125 \mathrm{~Hz}$ & $250 \mathrm{~Hz}$ & $500 \mathrm{~Hz}$ & $1000 \mathrm{~Hz}$ & $2000 \mathrm{~Hz}$ & $4000 \mathrm{~Hz}$ \\
\hline 8000 & $3.0 \pm 2.6$ & $5.6 \pm 1.9$ & $14.0 \pm 2.8$ & $20.4 \pm 2.5$ & $22.2 \pm 1.3$ & $22.6 \pm 1.1$ \\
4000 & $3.0 \pm 2.6$ & $5.9 \pm 1.9$ & $14.9 \pm 2.6$ & $21.2 \pm 1.9$ & $22.5 \pm 1.8$ & \\
2000 & $3.2 \pm 2.8$ & $6.7 \pm 1.9$ & $17.0 \pm 2.5$ & $22.7 \pm 2.4$ & & \\
1000 & $3.7 \pm 2.9$ & $8.5 \pm 1.8$ & $18.1 \pm 2.5$ & & & \\
500 & $5.0 \pm 3.3$ & $11.9 \pm 2.6$ & & & & \\
\hline
\end{tabular}

Table 2: Attenuation $[\mathrm{dB}]$ at select frequencies in lead V5 $(n=6)$.

\begin{tabular}{lcccccc}
\hline$F_{S}[\mathrm{~Hz}]$ & $125 \mathrm{~Hz}$ & $250 \mathrm{~Hz}$ & $500 \mathrm{~Hz}$ & $1000 \mathrm{~Hz}$ & $2000 \mathrm{~Hz}$ & $4000 \mathrm{~Hz}$ \\
\hline 8000 & $2.4 \pm 1.0$ & $5.1 \pm 2.4$ & $7.7 \pm 3.5$ & $12.9 \pm 6.2$ & $16.2 \pm 6.0$ & $18.2 \pm 4.6$ \\
4000 & $2.4 \pm 1.1$ & $5.4 \pm 2.6$ & $8.6 \pm 3.8$ & $13.9 \pm 5.8$ & $15.5 \pm 6.0$ & \\
2000 & $2.6 \pm 1.2$ & $6.2 \pm 2.8$ & $10.3 \pm 4.3$ & $14.6 \pm 6.5$ & & \\
1000 & $3.1 \pm 1.4$ & $7.9 \pm 3.3$ & $11.5 \pm 5.6$ & & & \\
500 & $4.4 \pm 1.6$ & $9.7 \pm 4.0$ & & & & \\
\hline
\end{tabular}

noise resulting from averaging. The noise term neglected in the mathematical treatment is present in the single beat spectra, but does not approach zero when the spectra are averaged. For the saECG case, the noise will indeed decrease in amplitude by a factor $\sqrt{N}$, where $N$ is the number of beats being averaged. In this small study, there was between 120 and 200 beats in each recording. Contributing further, the alignment of beats cannot be expected to occur within one sample for all beats. Hence, additional attenuation will occur from these beats. Finally, the underlying assumption, that all beats are identical, may not hold. Small differences between beats, both in timing and amplitude, will lead to a similar low-pass filtering effect as jitter. This effect could be the subject of further studies.

In conclusion, we found that the sample rate does influence the saECG spectrum; higher sample rates preserve more of the high frequencies, and therefore also likely preserve more information.

\section{References}

[1] Sörnmo L, Johansson ET, Simson MB. The Signal-Averaged Electrocardiogram. In: Macfarlane P, van Oosterom A, Pahlm O, Kligfield P, Janse M, Camm J. Comprehensive Electrocardiology. London: Springer, 2010: 1794-1821.

[2] Avitia RL, Reyna MA, Bravo-Zanoguera ME, Cetto LA. QRS complex duration enhancement as ventricular late potential indicator by signal-averaged ECG using timeamplitude alignments. Biomed Tech 2013; 58(2): 179-186.

Address for correspondence.

Jacob Melgaard

Aalborg University

Dept. of Health Science and Technology

Fredrik Bajers Vej 7, C1-214

DK-9220 Aalborg E

Denmark

jm@hst.aau.dk 
